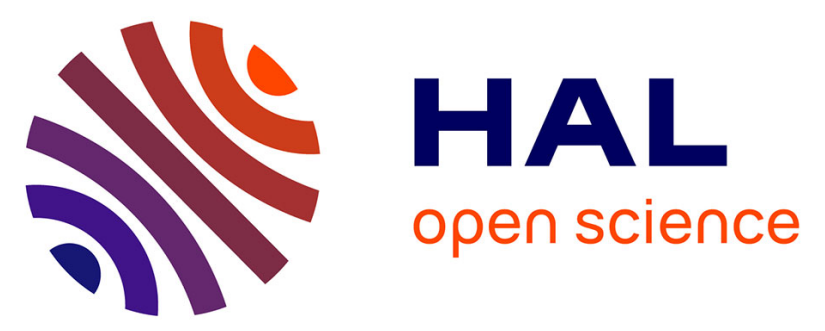

\title{
Les réseaux socialistes PSU en Bretagne (1959-1981): milieux partisans, passerelles vers le PS, rôle des chrétiens de gauche
}

François Prigent

\section{- To cite this version:}

François Prigent. Les réseaux socialistes PSU en Bretagne (1959-1981) : milieux partisans, passerelles vers le PS, rôle des chrétiens de gauche. Tudi Kernalegenn, François Prigent, Gilles Richard, Jacqueline Sainclivier (dir.). Le PSU vu d'en bas. Réseaux sociaux, mouvement politique, laboratoire d'idées (années 50 - années 80), Presses Universitaires de Rennes, pp.343, 2010. halshs-00444147

\author{
HAL Id: halshs-00444147 \\ https://shs.hal.science/halshs-00444147
}

Submitted on 6 Jan 2010

HAL is a multi-disciplinary open access archive for the deposit and dissemination of scientific research documents, whether they are published or not. The documents may come from teaching and research institutions in France or abroad, or from public or private research centers.
L'archive ouverte pluridisciplinaire HAL, est destinée au dépôt et à la diffusion de documents scientifiques de niveau recherche, publiés ou non, émanant des établissements d'enseignement et de recherche français ou étrangers, des laboratoires publics ou privés. 


\section{Les réseaux socialistes PSU en Bretagne (1958-1981) : milieux partisans, passerelles vers le PS, rôle des chrétiens de gauche}

Objet historique délaissé, décentré ou mythifié, les réseaux de la "petite gauche » du PSU s'avèrent une composante charnière aux contours mouvants dans la reconfiguration géopolitique des gauches en Bretagne entre 1958 et $2004^{1}$. Centrée sur les réseaux militants, cette étude recoupe une pluralité de sources compulsée dans le cadre de notre thèse en cours sur «Les réseaux socialistes en Bretagne au $\mathrm{XX}^{\mathrm{e}}$ siècle », sous la direction de Jacqueline Sainclivier. Les dossiers des Archives Départementales et du Centre d'Histoire du Travail de Nantes ont été confrontés au dépouillement systématique de la presse locale, des collections du Combat Socialiste et du Breton Socialiste (1958-1981), des fonds inexploités des archives fédérales du PS. L'examen des échantillons de sources annexes (PCF, CFDT, syndicalisme enseignant) et les fichiers des adhérents bretons SFIO-PS et PSA-PSU (listes intégrales des militants du PSU en 1958, 1965 et 1981), a été prolongé par une série d'entretiens ciblés auprès des élus et relais militants de la fraction socialiste, enrichis par la consultation d'abondantes archives privées. Cet article vise à saisir un milieu partisan hétérogène, articulant une somme de réseaux militants, au travers de la prosopographie de 3087 trajectoires d'adhérents bretons du PSU, mises en série dans une base de données, véritable « Maitron PSU Bretagne ${ }^{2}$ ».

L'implantation des réseaux PSU s'inscrit partiellement dans la continuité du milieu SFIO. L'emboîtement de deux vagues générationnelles (1958-1968, 1968-1975) ancre l'enracinement partisan autour de filières éclatées et imbriquées dans une multitude de myriades militantes. La méthode prosopographique dévoile une vue transversale statistique, cernant les profils militants et incitant à se pencher sur le multi-positionnement au sein de filières syndicales notamment. Le rôle décisif des chrétiens de gauche du PSU suppose de (re)définir les frontières de ces milieux pour interpréter cette dimension prépondérante de la conversion à gauche de la Bretagne, entamée entre 1958 et 1981 puis accélérée jusqu'au basculement de la séquence électorale 1981-2004. Pépinière militante en ébullition par l'apport novateur au niveau des idées, des filières d'adhésions, des interactions avec d'autres milieux, le PSU participe d'un renouvellement des générations à gauche, dont les recompositions perpétuelles dévoilent au final des PSU résolument pluriels. Magma de tendances difficilement réductibles à des sous-ensembles délimités, l'appartenance au PSU recouvre une hétérogénéité de références politiques allant du socialisme unitaire à l'auberge espagnole de la radicalité (autogestionnaire ou groupusculaire), qui bouscule les clivages identitaires de la gauche, à l'instar du rapport au marqueur originel de la laïcité. Parti socialiste transitoire et atypique, le PSU s'apparente en Bretagne à une passerelle politique, intégré par touches successives entre 1967 et 1982 à la refondation des réseaux socialistes. Matrice du socialisme version PS, l'aventure PSU est celle d'un lieu de passage qui influence les itinéraires militants.

\section{$\underline{\text { Jalons pour une prosopographie des militants PSU en Bretagne }}$}

\section{Spatialisation des réseaux PSU}

\footnotetext{
${ }^{1}$ PRIGENT F., «En haut à gauche ? L'implantation socialiste en Bretagne (1958-2008) au prisme de la reconfiguration des réseaux socialistes », journée d'études du Comité d'Histoire Politique Parlementaire, Paris, 30 janvier 2009 (à paraître).

2 Pour les études fines de trajectoires, voir les notices rédigées par nos soins. PENNETIER C., Maitron, Dictionnaire Biographique du Mouvement Ouvrier et du Mouvement Social, Paris, Ed. de l'Atelier, 2006-2012.
} 
Structure insaisissable et incomparable, la double hétérogénéité des nébuleuses PSU, dans les apports mêlés des provenances militantes et les divergences dans les directions/moments des sorties du parti, est signalée dans l'historiographie du PSU à l'échelle locale ${ }^{3}$. La singularité du bastion breton n'est plus à démontrer : mouvement politique solidement implanté durant les années 60 et 70 avec un volume total des sections bretonnes correspondant à $8-10 \%$ des adhérents nationaux, le PSU a aussi connu une transcription électorale ${ }^{4}$, près de $7 \%$ des militants identifiés ayant détenu un mandat sous l'étiquette PSA-PSU entre 1958 et 1977. Comptant 4 des 8 députés du parti (Tanguy Prigent et Yves Le Foll sont même les seuls représentants du PSU au parlement, respectivement en 1962 et 1973), la Bretagne fournit une cohorte substantielle de 22 conseillers généraux, 66 maires et 142 adjoints ou conseillers municipaux, principalement enracinés dans les Côtes-du-Nord et le Finistère. On dénombre aussi 3 anciens députés, 3 anciens conseillers généraux, 3 anciens conseillers d'arrondissement, 9 anciens maires, 22 anciens adjoints ou conseillers municipaux dans ce corpus pour lequel nous avons accumulé les notices biographiques. Pour autant, les fédérations bretonnes, mises en périphérie par l'absence de relais dans les cercles nationaux ${ }^{5}$, n'ont pas retenu outre mesure l'attention des dirigeants de premier plan, signe d'un parti qui se soucie moins de son impact en terme de mandats électifs que de la refonte des pratiques militantes ou du corpus idéologique du champ politique de la gauche. L'étonnant décalage entre l'identité militante éclatée du PSU national et la structuration plus homogène des fédérations départementales entraîne une rétraction sur l'espace régional des filières militantes PSU. Ce hiatus explique l'intégration au PS des infra-réseaux politiques du PSU, compris au sens de membres disposant de responsabilités électives. Fréquente dans le cas breton, la sélection de futurs cadres socialistes puisant dans le vivier de formation militante PSU donne à penser à un « accident historique de la famille socialiste 6 ".

Placées sous le signe des leaders, les lignes de force originelles du PSU dans les Côtes-duNord se prolongent dans le Trégor finistérien. En dehors de ces noyaux militants, la faiblesse électorale est contrebalancée par l'irruption dans les milieux urbains de groupes actifs extérieurs aux réseaux de gauche. L'approche fédérale comparative laisse l'empreinte des configurations différenciées de la genèse du PSU. Dans les Côtes-du-Nord, l'espace dissident des réseaux SFIO, qui maille le territoire dès 1936, processus renforcé après 1945, domine numériquement le PSU et invente une formule laboratoire à même d'intégrer des chrétiens de gauche et de nouer de nouvelles relations avec les communistes, comme à Saint-Brieuc en 1962. Dans le Finistère, la mouvance égocentrée qui suit Tanguy Prigent dans la scission du PSA, très localisée et marquée socialement, contraste avec la nébuleuse brestoise composée de chrétiens ouvriers et d'enseignants laïques (Roger Prat), souvent issus de l'Union de la Gauche Socialiste (UGS). En Ille-et-Vilaine, le milieu laïque polarisé à Rennes autour de

\footnotetext{
${ }^{3}$ SAINCLIVIER J., «Du PSU aux Assises du socialisme en Bretagne », in BOUGEARD C., Un siècle de socialismes en Bretagne (1905-2005), Rennes, PUR, 2008. MALFROY S. La fédération du PSU des Côtes-duNord face au Programme Commun. Visions et division de l'unité de la gauche, mémoire, IEP de Rennes, 2004. GUINEMENT J.L., La fédération PSU des Côtes-du-Nord, DES, Rennes 1, 1974. HARDEL S., Le PSU en Illeet-Vilaine de 1960 à 1990. Du parti de l'unité au parti de l'alternative, maîtrise, Rennes 2, 1997. GRAMOULLE G., Les origines du PSU en Cornouaille de la seconde guerre mondiale à la fin de la guerre d'Algérie, maîtrise, UBO, 1991. BOUGEARD C., Tanguy Prigent, paysan-ministre, Rennes, PUR, 2002. Voir les articles relatifs à la Bretagne dans cet ouvrage.

${ }^{4}$ En position de têtes de réseaux, une centaine de militants dirige et encadre des filières militantes de premier plan (organisations syndicales, milieu associatif et culturel, matrices chrétiennes, réseaux de jeunesse...).

${ }^{5}$ Rares bretons dans la Direction Politique Nationale, Tanguy Prigent et Antoine Mazier jouent un rôle dans la fondation du PSU, avec une complicité évidente avec Edouard Depreux, qui vient d'ailleurs à Saint-Brieuc pour le premier congrès fédéral du PSA en 1959. La résonance syndicale nationale des adhésions de Gilbert Declercq et Bernard Lambert est prise en compte lors du parachutage de Serge Mallet candidat à Rezé en juin 1968.

${ }^{6}$ En filigrane, l'expression de Michel Phlipponneau pose l'enjeu d'une histoire du PSU vue par les vainqueurs historiques du PS.
} 
Charles Foulon et Pierre Le Coadic, mâtiné par le poids des filières chrétiennes, est supplanté dans les années 68 par l'émergence d'un courant gauchiste, dont les Etudiants Socialistes Unifiés (ESU) constituent la fraction avancée. En Loire-Atlantique, la faiblesse initiale de la configuration initiale du PSA, menée à Nantes par Joseph Bercegeais, est relancée par l'entrée puissante des catholiques de gauche paysans et ouvriers (André Coutant, Maurice Milpied) qui transforment la physionomie du PS en 1975 avec l'entrée en bloc d'une composante militante soudée. Groupuscule bloqué, sans espace politique à l'ombre de la SFIO et du PCF, le PSU du Morbihan interpénètre des réseaux connexes influents : René Dervout (SNI), René Crouvizier (MLP-CGT), André Laurent (JOC-CFTC), Joseph Le Bouquin (CSF). La pondération des effectifs militants à partir des données d'adhésion par année, calcul difficile, ne permet pas de savoir exactement combien de Bretons sont passés par le PSU. Une estimation partielle, par recoupements à partir d'échantillons, situe probablement la réalité entre 4000 et 5000 individus au maximum (à comparer avec les 3087 parcours identifiés) ayant gravité dans la mouvance partisane PSU. La répartition départementale des effectifs donne à voir la spatialisation des réseaux militants : Côtes-du-Nord (32\%), Loire-Atlantique (30\%), Finistère $(18.5 \%)$, Ille-et-Vilaine (14\%), Morbihan (4.5\%), indéterminé $(1 \%)$.

La hiérarchie des pôles militants nuance la dissymétrie rural/urbain dans l'implantation du $\mathrm{PSU}^{7}$. La distribution des effectifs recensés, possible dans $93 \%$ des cas (2870 militants), est à comparer avec le nombre des adhérents par département ${ }^{8}$. La ventilation de $55 \%$ des militants se concentre au sein de 5 bastions privilégiés du PSU : le grand Nantes $(15 \%$, dont $10 \%$ pour la métropole), la couronne briochine (14\%, dont $10 \%$ pour la mairie qui incarne symboliquement la municipalisation des gestions PSU), Rennes (10\%), le Trégor morlaisien $(8 \%)$ et l'agglomération nazairienne ( $8 \%$ ). Dans un second temps, apparaissent des aires militantes qui pèsent pour $30 \%$ du total, sous la forme de noyaux militants compris entre 2 et $5 \%$ du groupe référence. Il s'agit de Rezé (5\%), des zones du Centre-Bretagne costarmoricain polarisées par Guingamp (4\%) et Loudéac $(4 \%)$, de Quimper $(3 \%)$, du Trégor (3\%), de la Cornouaille (3\%), des agglomérations brestoise $(3 \%)$, lorientaise $(2 \%)$ et vannetaise $(2 \%)$, ainsi que des secteurs de Paimpol-Tréguier $(2.5 \%)$ et Dinan (2\%). La dernière catégorie consiste en des archipels militants, micro-réseaux polarisés et isolés, dans la dorsale républicaine d'Ille-et-Vilaine $(1.5 \%)$, le Centre Finistère à dominante laïque (1.5 $\%)$, les régions de Châteaubriant (1\%), Saint-Malo (1\%), Fougères $(1 \%)$, le Sud Loire (1 $\%$ ), ou encore Douarnenez, Redon et Pontivy (1\% en cumulé), localisation imprécise (1\%).

\section{Les profils militants au PSU}

Pour brosser le portrait de groupe de ces réseaux militants, plusieurs trends sont à souligner sur la base d'un traitement statistique encore à perfectionner : âge, sexe, catégorie socioprofessionnelle, territoire, fonctions politiques, appartenance à des filières militantes...

\section{Une matrice de politisation de la jeunesse?}

La distribution par tranches d'âge, les données d'état civil étant connues pour $30 \%$ du corpus, est réalisée en prenant tour à tour comme date de référence 1960 (46 ans), 1965 (49 ans), 1968 (44 ans) et 1975 (42 ans). Rupture réelle entre les 2 âges du PSU, le moment 1968 dénote un profil médian d'une génération militante qui a 20 ans à la Libération. Nuançant les données nationales, biaisées par les jeunes noyaux militants parisiens, la composition générationnelle du PSU n'est pas très éloignée des autres milieux de gauches en Bretagne du $\mathrm{XX}^{\mathrm{e}}$ siècle, d'après nos études prosopographiques en cours. Le passé militant, acquis au sein de la base

\footnotetext{
${ }^{7}$ CONORD F., Les rets de la modernité. La gauche non communiste dans les campagnes françaises de la Libération aux années 1970, thèse, Université de Clermont-Ferrand, 2007.

${ }^{8} \mathrm{Cf}$. Annexes de l'ouvrage.
} 
SFIO, dénie l'idée d'un PSU formé aux origines de néo-militants ${ }^{9}$. La stabilité globale de ce profil durant la décennie 1958-1967 contraste nettement avec le nouveau cycle du PSU entre 1968-1972, véritable " passoire militante », qui rajeunit en profondeur ses cadres, préfigurant l'émergence de la génération qui acquiert des responsabilités politiques à partir de 1977 (2025 ans en 1968). Le renouvellement de plus du tiers des effectifs en 1968 répercute l'attraction sur la génération née après 1945. Au PSU, le flux d'adhésion, massif dans les villes, s'opère dans les sociabilités de jeunesse (étudiants, organisations de jeunes ruraux) : après 1967, un réseau de militants juvéniles (moins de 30 ans), sûrement minoré dans nos sources, saute aux yeux avec 86 ESU identifiés à Rennes et Nantes. Les parcours ultérieurs différencient les militants jeunes qui restent plus longtemps au PSU, attachés à des pratiques militantes radicales, avant d'évoluer ensuite vers l'extrême-gauche ou les Verts, et les militants au minimum trentenaires qui font carrière au $\mathrm{PS}^{10}$, avec une part non négligeable de transmission familiale.

\section{Un autre genre de parti ${ }^{11}$ ?}

On dénombre près de $1 / 6$ de militantes ( $14.4 \%$ avec 445 femmes identifiées), phénomène sous-estimé dans les archives, d'où ressort un noyau d'enseignantes (15\%), composé principalement des institutrices. Ces couples militants engagés s'accompagnent d'une prise de responsabilité syndicale ou associative. A l'avant-garde des réflexions sur la parité, le PSU se distingue des autres partis (PCF, MRP) qui opèrent par le haut une promotion féminine exceptionnelle. Conscient de l'atout de son attraction sur les milieux féminins, le PSU favorise l'ascension d'élues dans les réseaux secondaires (secrétaires de sections, candidates, élues). En regardant de plus près ces trajectoires, l'exclusivité des promotions des laïques, de façon indirecte par le biais de figures de l'autour dans l'ombre du mari/père ou dans l'entourage d'un notable, se double de parcours prolongés et accentués au PS, qui récupère et redéploie ces réseaux d'élues formées au PSU (aspirations des trajectoires de Marie Jacq, Marylise Lebranchu, Simone Darcel, voire Thérèse Caillaud). On observe donc une surreprésentation des adhésions préalables au PSU parmi le groupe des femmes PS, jusqu'à la féminisation de l'ère de la parité enclenchée dès le début des années 90. Les catégories populaires sont insérées dans les formes de militance familiale chrétienne, traditionnellement investie par les épouses/mères, Mouvement de Libération du Peuple (MLP), Confédération Syndicale des Familles (CSF). Les problèmes concrets de l'après-guerre, dont s'emparent en parallèle les structures avancées de l'Eglise (JOC), placent des femmes aux propriétés sociales indéfinies (normales) sur le devant de la scène. Dans la continuité de ces engagements multiples, un glissement vers la gauche paraît visibles dans certaines couches de ces réseaux, qui intègrent le PSU en deux temps : fort apport localisé Finistère/Loire-Atlantique/pôles urbains au moment de la préformation du PSU (1955-1961); flux plus ouvertement assumé dans une perspective féministe, concurrencée par les tendances d'extrême-gauche lors de la redéfinition politique hétérogène du PSU (1968-1972).

\footnotetext{
${ }^{9}$ La clé générationnelle, ressource mobilisée par le PSU, met en avant ces jeunes de moins de 40 ans au début des années 60 (responsabilités internes, candidatures locales). La notabilisation du PSU dans les années 70, qui se rétracte autour de réseaux d'élus locaux dans les Côtes-du-Nord et le Finistère renverse l'image nationale : le PSU vieillissant et institutionnalisé fait face à un PS rénovateur.

${ }^{10}$ Egalement des postes stratégiques de pouvoir dans les élites (économiques, universitaires, journalistes...).

${ }^{11}$ Une grille d'analyse du «genre du socialisme » en Bretagne a été proposée, in PRIGENT F., "Un autre genre de parti. Les femmes socialistes dans le Morbihan au $20^{\mathrm{e}}$ siècle », in Atala, $\mathrm{n}^{\circ} 11,2008$.
} 
Sociologie des militants PSU

\begin{tabular}{|l|l|l|}
\hline $\begin{array}{l}\text { Catégories socio- } \\
\text { professionnelles }\end{array}$ & $\begin{array}{l}\text { Trajectoires } \\
\text { identifiées }\end{array}$ & Proportion (\%) \\
\hline Professeurs & 231 & 16.1 \\
\hline Instituteurs & 214 & 14.9 \\
\hline Etudiants & 86 & 6 \\
\hline Cadres supérieurs & 155 & 10.8 \\
\hline Professions libérales & 79 & 5.5 \\
\hline Fonctionnaires/Employés & 168 & 11.7 \\
\hline Commerçants/Artisans & 97 & 6.8 \\
\hline Ouvriers & 184 & 12.8 \\
\hline Agriculteurs & 184 & 12.8 \\
\hline Mondes de la mer & 37 & 2.6 \\
\hline Professions connues & $\mathbf{1 4 3 5}$ & $\mathbf{4 6 . 5}$ \\
\hline $\begin{array}{l}\text { «Maitron PSU } \\
\text { Bretagne » }\end{array}$ & $\mathbf{3 0 8 7}$ & $\mathbf{1 0 0}$ \\
\hline
\end{tabular}

Avec 1435 professions connues dans l'échantillon, soit $46.5 \%$ du fichier total, les analyses par catégorie socioprofessionnelles montrent la prégnance des enseignants (445 dont 17.5\% de femmes), démultipliée dans l'encadrement partisan. Ce groupe s'impose avec $31 \%$ de l'échantillon (et $14.4 \%$ du dictionnaire complet de 3087 militants, dans la mesure où ils ont probablement été tous répertoriés). Occupant des positions internes fortes au PSU, les 231 professeurs ne masquent pas le réseau majoritaire d'instituteurs, investis dans les filières laïques, même si un glissement indéniable s'opère dans la direction des réseaux militants au profit des enseignants au plus fort capital culturel (notamment les universitaires). Les cadres supérieurs et professions libérales (234) forment $16.3 \%$ de l'échantillon ( $7.6 \%$ du total). La représentation sociale d'un parti de militants dotés d'un capital social + (pour reprendre une grille d'analyse bourdieusienne), sur-représentés de fait dans les sources, est visibilisée par cette prépondérance de $22 \%$ du volume total des militants repérés. Minorée, la proportion d'agriculteurs (184) atteint $12.8 \%$ (6\% du total), signe de la pénétration du PSU dans les espaces ruraux, plus affirmée après 1965. Pilier relativement faible du PSU, les 184 militants ouvriers (12.8\% de l'échantillon également) se répartissent à proportion égale entre militants chrétiens et laïques. Les catégories populaires avoisinent $12 \%$ de l'ensemble des militants recensés (le quart des professions connues). La catégorie fonctionnaires/employés (168 soit $11.8 \%$ du corpus) et des artisans/commerçants (97 soit $6.8 \%$ de l'échantillon) est conforme à la sociologie militante traditionnelle des structures socialistes, confortant la dimension « parti socialiste " du PSU. Ces analyses sociologiques accentuent le caractère transitoire du parti, qui récupère à la fois une partie du socle militant de la génération du Front Populaire de la SFIO et préfigure la recomposition du PS des années 70-80, initiée à la politique à partir des années 68.

Les fonctions politiques dont sont dotés ces militants cristallisent un groupe important d'élus ou de futurs élus (près de $14 \%$ ) : le PSU est un vivier, une école de formation politique, qui s'apparente à une matrice du PS, y compris en dehors des Côtes-du-Nord. L'existence d'un relais d'élus locaux sous l'étiquette PSU, transférés au PS en plusieurs vagues $(1967,1972,1975,1981)$, appuie les limites du PSU face aux débats internes ou aux 
controverses idéologiques, en décalage avec les configurations électorales d'une gauche non communiste à la recherche de nouveaux espaces politiques.

Le polyengagement accentué fait ressortir les enjeux cruciaux de l'investissement dans les filières syndicales de membres du PSU. Sur 307 militants dont l'appartenance syndicale est connue (en dehors des milieux enseignants), on repère une majorité de militants CFDT (52.8\%), engagés pour l'essentiel après 1967 . Une composante puissante reste syndiquée à la CGT (36\%), symbole de la position du PSU construite à la fois contre la SFIO et le PCF, tandis qu'un réseau FO résiduel $(11.2 \%)$ est hérité du courant socialiste. Ces données nuancent largement l'équation PSU=CFDT, qui ne prend pas en compte l'audience de la CGT (dont les espaces socialistes dissidents à l'instar de la trajectoire d'Albert Renouf à Rennes ou le choix de chrétiens engagés aux côtés des communistes, dans les noyaux de Nantes ou de Brest derrière Henri Berlivet), voire de la force de FO (dans certains milieux professionnels de la fonction publique à l'instar de Francis Mahéo ou Henriette Glon à Saint-Brieuc).

\section{Les filières enseignantes : poids et spécificités}

Legs de la SFIO (corrélation des taux), l'encadrement partisan par les mondes enseignants est aussi à lire en termes quantitatifs et symboliques, ces groupes sociaux pouvant accumuler des ressources politiques (capital militant) et mobiliser/polariser les réseaux militants. Les filières laïques sont le point d'entrée en dissidence du milieu socialiste, contestant les stratégies de troisième force et rejetant les choix de la ligne Mollet sur les enjeux coloniaux (la Guerre d'Algérie est un marqueur des réseaux PSU). Ces cadres partisans rompent plus facilement avec la SFIO que les élus ne comprenant pas le départ précipité et l'impréparation de la scission réalisée à marche forcée par Tanguy Prigent ou Antoine Mazier.

Surtout, la question syndicale enseignante s'apparente à un creuset, un modèle expérimental comme cela a pu être le cas dans les Côtes-du-Nord ${ }^{12}$. Dans un contexte marqué par la guerre d'Algérie, la remise en cause des lois laïques et le développement des luttes ouvrières, l'innovation Syndicat National des Instituteurs (SNI) débute en 1960 sous l'impulsion du SD communiste Maurice Renault, qui initie une liste d'union (bloquant jusqu'en 1978 l'émergence du courant Unité Indépendance Démocratie (UID) majoritaire au niveau national SNI-FEN) avec les dissidents de la SFIO regroupés dans le PSU, dont Sylvain Loguillard (UGS, laïque). Le bureau syndical hétérogène (1963-1969), dans la lignée de l'unité traditionnelle du syndicalisme départemental, remet en cause les divisions issues de l'autonomie de la FEN en 1948. Maurice Renault Unité Action (UA) et Yves Thomas Ecole Emancipée (EE), lui-même secondé par Louis Bocquet (directeur de la revue nationale EE, influencé par l'OCI), sont élus au Bureau National. Les militants PSU sont éparpillés entre deux tendances, le clivage se construisant sur la question religieuse. L'approche idéologique EE assimile la CFDT à l'appareil de la hiérarchie de l'Eglise dans le mouvement social, sans prendre en compte la révolution des pratiques militantes en cours dans les milieux chrétiens. Après 1968, l'EE opère une inversion de son analyse, appuyant la CFDT dans les conflits ouvriers locaux dans une stratégie gauchiste. Le PSU est une plate-forme agrégeant des sillons syndicaux différents, se retrouvant au SNI, lui-même laboratoire regroupant des militants venus d'horizons différents. Ces deux laboratoires ne se recoupent pas, travaillant sur des hypothèses différentes (PSU, autogestion; SNI union des partis de gauche). Le PSU s'apparente à un milieu bouillonnant où conflue différentes cultures. Comme dans les modèles en fractale, le PSU introduit ses agents dans un autre creuset (le SNI) dans lequel les questions identitaires se confrontent par le biais de problématiques professionnelles.

Les oppositions syndicales, non réductibles aux tendances internes du PSU, fixent le débat sur l'irruption de nouvelles pratiques militantes, préfigurant l'union de la gauche. Sur les bases

\footnotetext{
${ }^{12}$ PRIGENT A. (dir.), Des salles de classe aux luttes sociales. Mai-juin 1968 dans les Côtes-du-Nord, SaintBrieuc, Ed. FSU, 2009.
} 
d'un syndicalisme combatif imprégné d'une lecture sociale dominant/dominé, l'ouverture conjointe des milieux chrétiens et communistes au début des années 50 modifie les lignes de clivage avec des transferts militants dans les deux sens entre les deux pôles. Le PSU se positionne ici à l'intersection des mutations du champ social et politique ${ }^{13}$. Les frontières de la laïcité s'effacent au profit des pratiques unitaires, développant un discours revendicatif en direction des catégories populaires (vision du monde enseignant engagé en pointe du mouvement social). La rétraction des filières $\mathrm{EE}$ et $\mathrm{FO}$, crispées sur la question religieuse, conduit à une opposition interne au PSU sur les bases anticommuniste et anticléricale, reproduisant une alliance au centre prônée justement par l'ancienne SFIO, toujours honnie. Dans le même temps, la réunion des énergies entre chrétiens sociaux (dont une frange PSU) et communistes, associés aux espaces dissidents de la SFIO (donc PSU), révèle l'éclatement complexe des réseaux politiques du PSU. Cette expérience paradoxale d'une gestion conjointe des instances syndicales au sein d'un bureau unitaire (1963-1969) correspond à l'apogée du PSU dans les Côtes-du-Nord, qui se désagrège dans les années 70.

\section{Le PSU face au monde paysan, un modèle de réseau ouvert ${ }^{14}$}

L'univers paysan du PSU (12.8\% des effectifs) correspond à un double tissu, dont les formes de politisation datent de 2 périodes distinctes. Dans les années 58, les échos d'une implantation datant du Front Populaire, consolidés dans les territoires bretonnants depuis les luttes des années 30 (SFIO-Confédération Nationale Paysanne) et réactivés dans la Confédération Générale de l'Agriculture, se situent dans le sillon de la génération Tanguy Prigent (trajectoires des élus Yves Henry, Louis Le Mazier). Le passage au PSA-PSU du bloc paysan socialiste fait apparaître l'existence d'un réseau d'élus paysans laïques, souvent maires depuis 1945 ou 1947 (Louis Adam, François Charles), qui surplombe les sociabilités socialistes, conglomérat coopératif, syndical et associatif, à l'instar de l'itinéraire de Marcel Le Guyader. En dépit de cette densité militante localisée, le vote paysan échappe au PSU dans le reste de la Bretagne. La mutation est impulsée par un modèle de réseau ouvert, à savoir le réseau Lambert des années 68, étudié par ailleurs. Les liens partisans lâches, sans adhésion formelle, sont médiatisés par une figure syndicale, qui est elle membre du PSU. Sur le terrain gravite un réseau disparate, mobilisé sur des pratiques de luttes paysannes insérées dans un cadre bien plus large que le milieu partisan du PSU, porté par des filières contestataires chrétiennes (parcours typique de Jean Le Floc'h, JAC-CDJA). Les identités professionnelle et culturelle des paysans prennent le dessus sur la référence politique partisane, aboutissant à de nouvelles militances. Par le biais du PSU, ces courants sortent des espaces jusque là hermétiques à la gauche enfermée par le clivage laïque. La spécificité de ce réseau Lambert est la fréquence des syndicalistes proches du PSU. Fort en Loire-Atlantique, le phénomène est généralisé dans les autres départements où l'on repère des compagnons de route du PSU, soulevant la question du classement des "sympathisants PSU », mais qui ne franchissent le pas au PS qu'après 1972, dans la foulée des expériences syndicales de la rupture au sein de la FDSEA. Dans un contexte d'agitation sociale rurale, cette période trahit les tensions entre l'attraction idéologique tissée par le PSU, dans la foulée des analyses du colloque de novembre 1966 à Saint-Brieuc et de la diffusion du livre Les paysans contre le capitalisme après 1971, et les liens rattachant ces paysans au monde rural chrétien, mouvance dans laquelle cette génération s'est levée (associant des techniciens du monde agricole comme

\footnotetext{
${ }^{13}$ Le fonds CFDT confirme l'attractivité et la centralité du PSU. Les archives du PCF (fonds privé Marcel Alory, éditoriaux de la presse communiste) traduisent l'intérêt accordé aux mutations du PSU, partenaire vu comme un laboratoire de modernité. Il s'agit aussi d'une transformation du champ militant, moment de passage entre générations (1936-1958).

${ }^{14}$ Pour une réflexion théorique sur les modèles et typologies des réseaux politiques. Prigent F. (dir), « Les réseaux socialistes dans le Morbihan au $20^{\mathrm{e}}$ siècle », in Recherche Socialiste, $\mathrm{n}^{\circ} 42,2008$.
} 
Jean-Baptiste Henry). Passeur politique, le PSU participe de la fabrique des futurs cadres paysans du PS, accédant aux mandats d'élus dans les années 80. Ces carrières politiques sont lancées, dans/autour/grâce/à cause le PSU (Louis Chopier, Bernard et Maurice Thareau, Joseph Guénanten...).

\section{Le rôle des chrétiens de gauche du PSU}

Les rapports entre socialisme et religion se redéfinissent dans la deuxième moitié du $\mathrm{XX}^{\mathrm{e}}$ siècle, notamment après le concile de Vatican II, transfigurant le champ politique régional. Parmi les acteurs de l'inversion de la religion, aux côtés des réseaux CFDT, le PSU rassemble une nébuleuse de structures militantes composites, évoluant dans les sociabilités chrétiennes progressistes en phase de décrochage avec le bloc conservateur dominant.

Le profil de ces sous-ensembles militants du PSU appelle plusieurs remarques liminaires. L'interrogation de ces réseaux se heurte à des écueils d'ordre quantitatif et qualitatif, en raison de la nature même des sources, qui rend complexe l'identification du processus de dérive à gauche de ces franges militantes et des réseaux qui gravitent autour. L'influence réelle de ces noyaux militants à rapprocher d'autres groupes de référence de volume limité qui suivent une évolution similaire. Les étiquettes de "chrétiens », "chrétiens de gauche », "chrétiens du PSU » ne doivent pas être cloisonnées pour comprendre un panel de comportements dont les mutations sont progressives : fin du vote conservateur à dominante cléricale, distanciation avec la pratique religieuse, rupture dans les fréquentations de réseaux sociaux polarisés par la religion, réflexion sur la laïcité et redéfinition du rapport à l'école, rapprochement des organisations de gauche. Une réflexion méthodologique est encore à mener, autorisant les démarches comparatives à partir d'études de cas prosopographiques, pour donner du sens à ces profils de militants doubles, qui refaçonnent l'empreinte religieuse de la société bretonne $\mathrm{du} \mathrm{XX}^{\mathrm{e}}$ siècle. Les itinéraires (voies, modalités, causes) de séparation/éloignement des formes traditionnelles de l'Eglise remettent en cause l'homogénéité du milieu des «cathos de gauche $»$, reconstruite a posteriori ${ }^{15}$ ? Le PSU procède de cette lame de fond qui submerge et transforme l'Ouest breton, et la renforce. Le concept de réseaux, mis en perspective avec les notions de champ partisan, trajectoires militantes, identités politiques, se heurte aux limites de la représentation graphique des aires d'interrelations militantes. Les interactions entre ces micro-réseaux et le milieu PSU, voire d'autres milieux politiques, sociaux sont à creuser. Lees leaders de ces réseaux égocentrés (représentatifs de ces infra-réseaux militants) sont parties prenantes de l'attraction de la gauche sur des milieux extérieurs au PSU. L'ensemble de ces interrogations méthodologiques, conceptuelles, analytiques est obscurci par l'éclatement d'archives inconnues et réduites, encore en cours d'exploitation. Cette vision parcellaire, fragmentée de l'historien interfère avec le schéma mental dominant, véhiculé par les acteurs eux-mêmes, qui postule l'existence d'un milieu de "cathos de gauche» uni, alors que l'appellation recouvre des réalités très diverses, dont le transfert au PS des élites militantes, au terme d'un cheminement qui bascule lors d'une expérience PSU jugée fondatrice au même titre que les passages par la JOC ou la JAC, a révolutionné en Bretagne le visage de la gauche ouverte et conquérante dans les années 70-80. L'histoire de ce mouvement reste encore à démontrer par l'intermédiaire d'études de cas étayées.

Les matrices chrétiennes du PSU couvrent $7 \%$ des effectifs militants repérés en Bretagne, soit 216 trajectoires identifiées formellement, cette base représentative resserrée relativisant la portée des analyses qui suivent. Ces militants investissent des réseaux étroits et imbriqués, mais la surface d'influence de ces milieux dépasse sa faiblesse numérique car les pratiques militantes de ces réseaux déplacent à gauche des forces pivots grâce à un rayonnement par

\footnotetext{
${ }^{15}$ DONEGANI J.M., «Itinéraire politique et cheminement religieux : l'exemple des catholiques militants du PS », in Revue Française de Science Politique, vol. 29, n4, 1979.
} 
capillarité sociale qui diffuse des valeurs politisées, à partir des liens tissés dans une multitude d'entourages. Les filières CFTC forment le premier cercle matriciel, presque systématique (2/3 des itinéraires), agglomérant des parcours décisifs dans la prise de conscience générationnelle, sous l'influence conjuguée de la guerre d'Algérie et des nouvelles formes de la question sociale, porteurs d'un syndicalisme de terrain qui reste l'identité militante la plus valorisée. Les rapprochements parallèles avec la gauche (CFDT, PSU, PS) sont accélérés par la forte présence de l'encadrement syndical des UD au PSU : Gilbert Declerq, André Laurent, André Marivin, Jean Le Faucheur, Michel Cadoret ${ }^{16} \ldots$

Les réseaux politiques, existant sous différentes formes (JR, MRP, UCP, UGS, NG), sont très présents dans les parcours de ces militants (62\% des cas). Il s'agit d'un primo-militantisme dans des réseaux politiques, prolongeant l'engagement dans des réseaux plus concrets, qui finissent pas fusionner dans l'UGS, avant d'intégrer en bloc le PSU (Pierre Bourges, Janine Palm). Il existe une sur-représentation de ces réseaux, dans les espaces de faible ancrage du PSU, dont le Morbihan, ou dans les territoires urbains. Cette dimension doit être rapportée à la fréquence des passages préalables par les milieux radicaux mendésistes (CAD), comme Pierre Sérandour, ou le PCF en Ille-et-Vilaine par exemple. L'adhésion au PSU n'est pas la découverte du militantisme politique pour les catholiques, tandis que les engagements individuels et dispersés dans la JR dès les années 1930, puis au MRP dans les années 40 et l'Union des Chrétiens Progressistes (UCP) au début des années 50, prolongent une militance chrétienne sociale, notamment pour les milieux populaires MLP, CSF, Action Catholique Ouvrière $(\mathrm{ACO})^{17}$ et traduisent un processus lent de rupture avec les structures officielles de l'Eglise. Ces mutations, accordant une place particulière dans la culture politique au volet de l'action sociale (refus de l'injustice) s'accompagne d'un déplacement du vote vers la gauche, d'une multiplicité d'investissement dans des réseaux chrétiens sociaux et de l'adhésion à de petits groupes bouillonnants à l'instar de NG après 1955, particulièrement dans le Finistère. Ces structures, qui posent des liens bilatéraux entre le militant et le réseau, sont fondues dans l'UGS après 1957 avec une mixité culturelle au vu de l'accueil de trajectoires venues de la gauche du socialisme. Point d'aboutissement d'un déplacement vers la gauche de petits réseaux personnels, l'UGS est une filière d'intégration du PSU, dont il ne faut pas négliger l'influence. Ces sillons politisés s'accompagnent d'une syndicalisation à la CGT dans les pôles urbains, tout en étant vecteur d'un engagement catholique social laïcisé (Jacques Galaup).

Le passage marquant par les organisations de jeunesse (17.6\% des cas) révèle le rôle décisif de structures militantes qui ne sont pas ancrées à gauche de façon monolithique, comme la JEC et la JOC. La sous-représentation de ces catégories dans les sources, au regard du continuum des sympathisants PSU futurs socialistes à partir des années 70 , fait apparaître un vivier important de cadres PS, marqués par une contestation générationnelle forte. Ces milieux jocistes turbulents et ouverts, forgés durant l'après-guerre, s'autonomisent par rapport à la hiérarchie catholique et se rapprochent du champ communiste. De façon logique, ces militants qui ne peuvent aller ni vers le PCF, ni retourner vers l'Eglise se retrouvent temporairement au PSU, faisant fonction de réseau de transition politique dans une évolution globale de ces milieux chrétiens qui intègrent la gauche socialiste depuis les années 70 .

\footnotetext{
${ }^{16}$ L'approche sur le long terme des réseaux socialistes de la CFDT est une piste de recherche en cours.

17 Traduction d'une politisation du quotidien, l'univers associatif chrétien laisse une empreinte fondatrice, à la hauteur de la force religieuse de l'ancrage dans le réel de ces groupes. Par le changement des pratiques (plus collectives, plus émancipées) et l'imprégnation de valeurs humanistes prenant un sens nouveau dans un contexte social transformé. Ces réseaux associés à la hiérarchie conservatrice catholique dérivent en partie vers la gauche (le PSU étant un parti moins repoussant pour les chrétiens). Cette évolution qui neutralise l'enjeu politique de la religion se fait au PSU, dernière étape avant la sortie du giron de l'Eglise.
} 
Les réseaux ruraux (JAC, CDJA, MRJC, FDSEA) forment moins de 15\% de ce corpus, certainement minoré dans les sources, d'autant qu'on observe une distorsion entre la faiblesse de ce type de trajectoires au PSU et l'influence de ces parcours sur le vote paysan en faveur du PSU, capté ensuite par le PS. Moment de passage fondamental dans les trajectoires PSU des futurs élus socialistes (René Philippot), cette politisation en milieu rural, qui éloigne les jeunes agriculteurs de la domination conservatrice, prend des formes d'adhésion en pointillés. Dans les études fines de parcours individuels, l'investissement ou la connivence avec des réseaux intellectuels à dimension spirituelle (Témoignage Chrétien, La Vie Nouvelle) pèsent pour moins de $5 \%$ des effectifs. La fonction essentielle de ces réseaux, cimentés par l'ACO, est de tisser des liens avec un clergé progressiste (cercles religieux, prêtres-ouvriers, expériences dans l'Eglise) : le rôle des rencontres humaines dans un cadre spirituel s'avère un élément déclencheur et une ligne de clivage dans la réorientation politique de ces militants, initiés dans le PSU. Démarches individuelles, ces tranches de vie sont difficilement transposables pour retracer un faisceau de comportements.

Les réseaux de la militance familiale, qui imprègnent les milieux populaires urbains, sont multiformes (MLO-MLF-MLP, CSF-APF, voire scoutisme) et concernent plus de $20 \%$ des militants chrétiens du PSU. Volet mal connu de 1'histoire sociale bretonne, ces réseaux réunissent des mini-structures locales, sans unification, forgées au moment de la reconstruction en 1945. Emanations des filières ACO, ces réseaux d'inter-connaissances sont fortement implantés sur la façade Ouest de la Bretagne, alors que la SFIO voire dans une moindre mesure le PCF ne mordent pas sur ce type de milieux ouvriers. Paradoxalement, ces réseaux qui semblent négligeables quantitativement jouent un rôle décisif dans le glissement de ces franges militantes, par capillarité.

Minoritaires dans le PSU, l'approche de type microstoria des trajectoires de sociaux-chrétiens confirme le rôle des réseaux PSU dans les transferts géopolitiques entre le MRP et le PS (1945-1981). Esquissée lors de la communication orale, il faudrait élaborer une grille de lecture conceptualisée pour saisir ces mutations d'un monde qui s'en va, à savoir les effets du délitement du champ religieux moderne sur le champ sociopolitique. Parti sécularisé qui s'ouvre aux filières chrétiennes, le PSU est un pivot qui reflète les réalités du changement politico-religieux multiples selon les dimensions observées (ouverture, inversion, degré). Les schémas explicatifs (comment, pourquoi, quels déterminants ?) de ces passages vers la gauche nécessiteraient de mesurer et de représenter plus globalement les liens entre religion et politique.

\section{Les passerelles socialistes : l'influence du PSU sur le socialisme version PS}

Matrice irriguant le ressourcement du recrutement du PS, agrégation de réseaux à bases multiples dans les années 70, la part du courant PSU incontestable dans la pesée militante globale met en exergue la promotion d'ex-PSU dans les «élites roses de la génération Mitterrand ».

\section{Mythe ou réalités ?}

Rassemblement de ramifications dissidentes, le PSU né entre deux cycles socialistes connait une triple vague de transferts vers la nouvelle structure partisane du PS. Entrée en militance sur des débats et poussée hors du PSU par l'absence de débouchés électoraux, une fraction des socialistes élus depuis 1971 a quitté les divisions internes des groupuscules PSU entre 1967 et 1972. L'essentiel des ex-PSU reconvertis au PS adhère dans la foulée des Assises en 1974-1975 individuellement, en bloc ou par réseaux fragmentés. L'ouverture de l'affiliation socialiste suscite des tensions attisées par l'absence de fusion des matrices qui confluent au 
$\mathrm{PS}^{18}$. Le flux tardif d'adhésions dans les années 80 conclue à un passage PSU/PS dont le taux est supérieur à $80 \%$, donnant une teinte caractéristique à la social-démocratie d'élus locaux du PS en Bretagne, dont la maturation prend fin en 2004.

Les entretiens réalisés avec les élus PS depuis 1968 surévaluent la dimension du PSU, plateforme de décrochage du bloc conservateur des organisations chrétiennes de jeunesse (JACJOC) par proximité avec les luttes sociales, point de départ de l'irruption d'un nouvel encadrement partisan au début des années 70. La mention systématique "sympathisants PSU » dans les témoignages recueillis est significative de cette vision mythifiée du PSU. La dimension matricielle du PSU dans les racines composites du PS se mesure par régression, en comparant les trajectoires spécifiques au PS des anciens du PSU. Au-delà de l'échelonnement discriminant dans le temps des départs, la puissance de l'héritage et la similitude des itinéraires font penser à une strate PSU exportée au PS. Le phénomène est amplifié pour le corpus des élus, le PSU jouant le rôle d'école de formation et de référence idéologique première dans les trajectoires. Sur les 3087 militants identifiés, on recense plus de $5 \%$ d'élus sous l'étiquette PS entre 1975 et 2008: 21 parlementaires, 39 conseillers généraux, 11 conseillers régionaux, 43 maires, 75 adjoints ou conseillers municipaux ${ }^{19}$.

\section{Militance tremplin, lieu de formation politique}

Indéniable lieu d'acquisition d'un capital militant, l'empreinte PSU sur les têtes de réseaux du courant rocardien active des filières homogènes, partageant une formation commune: l'expérience PSU de ces transfuges, insérés dans des milieux construits à côté des contresociétés communiste, socialiste voire chrétienne. Par un déplacement minime de voix au final (inférieur à $5 \%$ ), le PSU a transféré une partie de son enracinement multiforme au PS, recentré par la bipolarisation de la vie politique bretonne, qui marginalise les autres votes ${ }^{20}$.

Cette configuration d'un moment PSU, entre-deux des réseaux socialistes, est représentative des parcours d'élus PS, aguerris politiquement au PSU et investis dans des réseaux militants qui dynamisent les gauches. La prime à l'ancienneté dans le PS se mesure en mois pour ces jeunes figures socialistes sortis du PSU dans les années 1968 après une courte adhésion, profitant d'un vide circonstanciel pour accaparer les places dans un PS embryonnaire. Les secondes dissidences de Claude Saunier, Marie Jacq, Roland Andrieu, Pierre Jagoret ou Georges Cano (des filières Poperen en 1967 à la FGDS et au courant post Epinay) précèdent l'expansion électorale du PS dans un grand Ouest, réservoir de voix pour la gauche non communiste à l'échelle nationale. La fermeture des fenêtres de pouvoir dans les réseaux dirigeants après 1973 retarde les parcours de la majorité qui intègre le PS en 1975 seulement, à l'exception des principaux leaders rocardiens. A partir de 1971, les étiquettes persistent, empêchant la fusion des réseaux qui se segmentent et se concurrencent à l'intérieur des espaces partisans : la filière d'origine et la date d'adhésion déterminent les positionnements dans la hiérarchie interne, avec un maintien de réseaux socialistes parallèles, tenant des réunions séparées et faisant resurgir des solidarités PSU passées. Les oppositions interpersonnelles reproduisent les luttes des années 60 (SFIO/PSU) dans les nouvelles divisions de tendances des années 70 (CIR/CERES/PSU...), très ancrées dans le terreau politique local. Les anciens PSU extérieurs aux conflits internes et dotés d'une expérience nationale sont propulsés dans les cercles dirigeants du PS (Didier Chouat). Parmi les figures socialistes émergentes des années 90 , la part de la transmission familiale est présente (Thierry

\footnotetext{
${ }^{18}$ Réfractaires, opposants, indifférents : l'hostilité contre «l'œil de Rome et la gauche américaine » est une attitude prégnante au sein du PS.

${ }^{19}$ Plusieurs enquêtes empiriques au cœur du PS montrent un passage par le PSU de $15 \%$ de la base miliante.

${ }^{20}$ La plasticité des réseaux socialistes, dont la longévité séculaire est remarquable dans le système partisan, est le produit d'un empilement de votes catalysés: radicaux après 1945, chrétiens démocrates après 1968, communistes après 1989.
} 
Troël), processus couplé à l'aspiration des filières CFDT et à la mise en place d'un sérail de renouvellement des réseaux par les experts socialistes professionnalisés (attachés parlementaires, cabinets ${ }^{21}$ ).

Le rayonnement autour de marges sympathisantes au statut presque reconnu et associé au milieu partisan montre la séduction idéologique du parti qui se démarque du modèle militant communiste dominant à gauche. Traces résiduelles d'une fascination pour le PSU, les compagnons de route soulignent l'arrimage des réseaux PSU aux luttes sociales des années 68 en Bretagne, contestation régionale aussi portée par la CFDT ou les héritiers de la JAC. Le projet régional du PS puise et recycle les idées élaborées par les configurations partisanes du PSU, qui inspire les postures des dirigeants socialistes. Frappantes, la structuration régionale des réseaux socialistes au sein du Bureau Régional des Etudes et Informations Socialistes (BREIS) et l'assimilation des thèmes privilégiés et novateurs du PSU sont des pratiques impulsées de façon concomitante par d'autres réseaux que le PSU Bretagne (organe de presse communiste, UR CFDT, réunions régionales SFIO-FGDS). La régionalisation du socialisme breton, incarnée par les trajectoires charismatiques de Louis Le Pensec et Charles Josselin, procède directement de la rencontre idéologique et humaine de deux courants, qui s'entrechoquent dans le nouveau réseau d'élus, en écho au thème du colloque de Saint-Brieuc en novembre 1966, « Décoloniser la Province ».

\section{Les vagues de départs}

L'absorption du PSU dans le PS ne se réduit pas au courant national en octobre 1974 des Assises du Socialisme ${ }^{22}$, mais plutôt à une série de départs discontinus vers le PS. En 1967, les proches de Poperen quittent le PSU avant l'afflux massif de 1968 en prônant l'union de la gauche, dont le PSU est plus une force d'appoint qu'un pivot. En 1972, le rejet des positions gauchisantes de la frange activiste du PSU déstabilise la base militante (entrées volatiles et sorties des cadres). Les Assises, comme lors de la première scission (1958-1960), sont mal préparées et incomprises localement. Dans les Côtes-du-Nord, comme dans le Finistère, il existe 2 courants séparés depuis 1972 : la tendance gauchiste majoritaire (André Le Milinaire) s'oppose au groupe des élus (pôle briochin, conseillers généraux, maires ruraux) qui apporte au PS une assise électorale. La direction des Assises (140 militants), derrière Yves Dollo, entre au PS au terme d'un vote très serré, emportant l'organe de presse et le poids financier. Mais les ex-PSU se retrouvent bloqués, en dehors des cercles rocardiens, dans une fédération éclatée, en guerre ouverte. Autre spécificité, le passage des cadres principaux de la CFDT (Jean Le Faucheur, Michel Cadoret) est mûrement réfléchi, ayant déjà quitté leurs responsabilités syndicales pour des mandats politiques. En Loire-Atlantique, la majorité passe en bloc au PS malgré la division des militants du PSU, incarnées par leurs leaders. Claude Evin structure une force au profil militant proche, amalgamant les matrices JOC, CFDT, PSU, alors que François Autain choisit l'ancrage du CERES, sacrifiant le brouillage des frontières de la laïcité (présence du groupe Guy Goureaux du Cercle Jean XXIII) aux impératifs d'un ancrage à gauche (stratégie unitaire aux côtés du PCF). La question des relais syndicaux est ici partiellement réglée avec l'entrée en groupe des paysans syndicalistes au PS dès 1972, mais aussi le passage précoce au PS de l'entourage CFDT de Gilbert Declercq. En Ille-etVilaine, la négociation entre Pierre-Yves Heurtin (figure du nouveau PSU façonné après 1968) et Edmond Hervé (élu conseiller général en 1973, qui a capté un réseau d'élus, comme Albert Dory, Georges Cano ou Jacques Faucheux, passés par le PSU) aboutit à une entrée par petits paquets de plus des trois quarts des adhérents PSU. Sans stratégie de coordination, la concurrence antagoniste avec le CERES autogestionnaire de Jean-Michel Boucheron oblige à

\footnotetext{
${ }^{21}$ SAWICKI F. et LEFEVRE R., La société des socialistes, Ed. du Croquant, 2006.

${ }^{22}$ KRAUS F., Les assises du socialisme ou l'échec d'une tentative de rénovation d'un parti (octobre 1974), Paris, Notes de la Fondation Jean Jaurès, n³1, 2002.
} 
une alliance forcée avec les réseaux CIR en déclin de Michel Phlipponneau, tandis que le malaise désordonné des PSU-CFDT est marqué par la trajectoire d'André Marivin, signataire des Assises qui diffère finalement son adhésion. Dans le Morbihan, il n'existe pas à proprement parler de mouvement des Assises. Le groupe PSU a suivi une direction alternative (nourrissant les réseaux trotskiste, maoïste, écologiste) avec la particularité d'un désengagement politisé après la sortie du PSU (René Le Pauder). Les chrétiens du PSU ne sont pas destinataires de l'ouverture de la gauche socialiste, spectaculaire en 1971 sur la liste Yves Allainmat à Lorient auprès de réseaux localisés et associatifs cimentés par la JOC, prolongée ensuite par l'ascension de Jean-Yves Le Drian qui catalyse ces énergies depuis sa victoire aux législatives de 1978. L'entrée au PS des chrétiens de gauche se fait sur une courte décennie (1970-1979) dans la région de Vannes, avant d'être significative à partir des années 90 où les communes conservatrices se dotent d'élus puisés dans les filières laïques et dans des individus détachés du bloc chrétien, parfois par la piste PSU, qui ne semble pas ici déterminante ${ }^{23}$.

La part des militants PSU est à replacer dans un questionnement englobant du système partisan. Angle mort de l'historiographie, ces micro-réseaux mobilisent des milieux militants à bases multiples, fonctionnant sur le modèle des partis-mouvements qui rayonnent en fédérant des réseaux associés. L'inversion de l'inscription des filières de chrétiens de gauche, qui échappent à la SFIO et au PCF en transitant par le PSU, contribue à l'émergence d'une gauche non-communiste depuis 1945. Si les instruments de mesure des transferts électoraux restent à affiner, au même titre que l'histoire balbutiante de ces nébuleuses militantes, le renouvellement du système partisan procède aussi de l'irruption à gauche de réseaux militants aux ancrages nouveaux qui cultivent le bouillonnement politique de l'éphémère PSU.

Histoire du temps présent privée d'approches monographiques locales, ces recompositions partisanes invitent à repenser la périodisation. L'effet combiné de la révolution du pouvoir gaulliste et du choc de la Guerre d'Algérie positionne 1958 en une césure incontournable dans le second $20^{\mathrm{e}}$ siècle. Evoquée à l'échelle nationale, la réalité des années 68 cristallise une série de mutations lentes, qui bouleverse les organisations de gauche tissant de nouveaux liens avec le mouvement social par le biais de filières militantes ouvertes. Les départs du PSU, ses transformations internes entre 1967 et 1972 sont des années « tournant » pour une mouvance politique atypique, dont la portée se mesure aussi à l'aune des devenirs militants de ses membres. Dans le courant rocardien, les ex-PSU impulsent une culture politique nouvelle au PS, dont les effets impriment l'émergence d'une génération de cadres socialistes au même titre que le souffle de rénovation des Clubs ou du CERES. Dominante, la frange socialiste du PSU s'oppose au glissement d'une autre génération militante vers une culture alternative et radicale, active dans l'écologie politique et les milieux d'extrême-gauche. La rupture des municipales de 1977, seuil électoral qui sanctionne les poussées du cycle d'Epinay, entamé en 1973 et clôt en 1982, démontre la dissolution majoritaire par transferts dans le milieu socialiste du PSU.

Au-delà des enjeux de la démarche prosopographique ${ }^{24}$, cette étude croise les analyses de trajectoires dans un portrait collectif des réseaux militants du PSU. La diversité des profils et

\footnotetext{
${ }^{23}$ La question du PSU resurgit en réaction à l'entrée massive des chrétiens de gauche dans un parti jusque là implanté sur des contre-sociétés läques, aboutissant à la prise de contrôle des Rocardiens en 1977 (Joseph Le Lamer ancien proche du PSU et Philippe Meyer investi dans les GAM d'émanation idéologique PSU). L'affaire du parrainage Bouchardeau par Jean Giovannelli est un prétexte qui ouvre une guerre intestine, source de déstabilisation des socialistes du Morbihan: exclusion du nouveau député, dissolution de la fédération et tarissement des adhésions.

24 PRIGENT F., «Des fiches aux fichiers. Les réseaux socialistes dans l'Ouest breton des années 1930 aux années 1980 : archives, trajectoires, prosopographie ", communication à la journée d'étude du CERHIO (Le Mans, 12 avril 2008), à paraître.
} 
la multiplicité des filières imbriquées/milieux investis fondent la réalité du laboratoire politique PSU. Les aires militantes, dessinées à partir de 3087 parcours biographiques, assurent la connaissance de l'enracinement des réseaux du PSU. Seulement esquissé, il reste à creuser le modèle interprétatif des réseaux spécifiques chrétiens de gauche, dont l'intégration à gauche contribue à nourrir l'apparition de puissants réseaux socialistes. L'héritage PSU dans l'histoire récente du PS en Bretagne dépasse ses apports au niveau de la culture politique : les formes d'influence dans les devenirs militants montrent la force du réservoir PSU, vivier de cadres socialistes et lieu d'invention de pratiques militantes, de schémas idéologiques pour les " menhirs socialistes bretons », génération homogène qui entre en politique dans les années 68 et accède aux responsabilités entre 1981 et 2004. Composante centrale du PS, le PSU est une double filière socialiste : les origines du «parti passoire » fournissent un cadre original à même de rassembler des courants qui se fondent provisoirement dans les visages successifs du PSU; les sorties du "parti passerelle » aux âges multiples participent de la relance de la gauche socialiste, qui rencontre dans les années 70 un contexte favorable à l'élaboration d'une force politique dominante, voire hégémonique en Bretagne. 